\title{
Postoperative care after lymphaticovenous anastomosis
}

\author{
Jeffrey C. Y. Chan ${ }^{1}$, Giuseppe Di Taranto ${ }^{1,2}$, Rossella Elia ${ }^{1,3}$, Vittoria Amorosi ${ }^{1,4}$, \\ Ngamcherd Sitpahul ${ }^{1,5}$, Hung-Chi Chen ${ }^{1}$ \\ ${ }^{1}$ Department of Plastic Surgery, China Medical University Hospital, Taichung City, Taiwan; ${ }^{2}$ Department of Plastic and Reconstructive \\ Surgery, Umberto I University Hospital, Sapienza University of Rome, Rome; ${ }^{3}$ Division of Plastic and Reconstructive Surgery, Department of \\ Emergency and Organ Transplantation, University of Bari, Bari; ${ }^{4}$ Plastic Surgery Unit, Sant'Andrea Hospital, School of Medicine and \\ Psychology, Sapienza University of Rome, Rome, Italy; ${ }^{5}$ Department of Plastic and Maxillofacial Surgery, Faculty of Medicine, Ramathibodi \\ Hospital, Mahidol University, Bangkok, Thailand
}

In this report, we discuss the postoperative protocol for patients undergoing lymphaticovenous anastomosis (LVA) in our unit. Immediately after LVA, the incision site is closed over a small Penrose drain and a simple gauze dressing is applied without compression. In the first 5 days, ambulation is allowed, but limb elevation is actively encouraged to promote lymphatic flow across the newly formed anastomosis. Prophylactic antibiotics are routinely given to prevent infection because this patient group is susceptible to infections, which could trigger thrombosis in the anastomosis.

Keywords Lymphaticovenous anastomosis / Lymphedema / Postoperative care / Compression

\author{
Correspondence: Hung-Chi Chen \\ Department of Plastic Surgery, China \\ Medical University Hospital, 2 Yuh- \\ Der Road, Taichung City, 40447 \\ Taiwan \\ Tel: +886-4-2205-2121 \\ Fax: +886-4-2202-0038 \\ E-mail:D19722@mail.cmuh.org.tw
}

\section{INTRODUCTION}

Lymphaticovenous anastomosis (LVA) is a popular procedure performed at many centers in the world as a surgical solution for lymphedema when conservative management is inadequate. The reasons for its high acceptability among patients include its minimally invasive nature, as well as the fact that it can be performed under local anesthesia and patients can be treated on an outpatient basis. The short-term results of LVA are promising, although evidence for its long-term efficacy has not been firmly established [1].

At many lymphedema centers, the operated limb is compressed with a pressure garment immediately after surgery, and the patient is discharged home on the same day [2]. Recent publications based on intraoperative observations have suggest- ed that immediate limb compression distal to the anastomosis site promotes lymphatic flow across the anastomosis, which is thought to maintain its patency $[3,4]$. However, no medium- or long-term studies are available to support this practice. In our unit, we follow a more cautious approach to the postoperative care of patients after LVA procedures.

\section{CASE}

Herein, we describe the case of a 76-year-old woman with bilateral lower limb lymphedema (Fig. 1). Her previous medical history included atrial fibrillation, hypertension, stroke, hypothyroidism, and renal insufficiency. She presented with recurrent paronychia and cellulitis on her left lower limb over the past 4 to 5 years. Limb swelling progressed over this time period and 


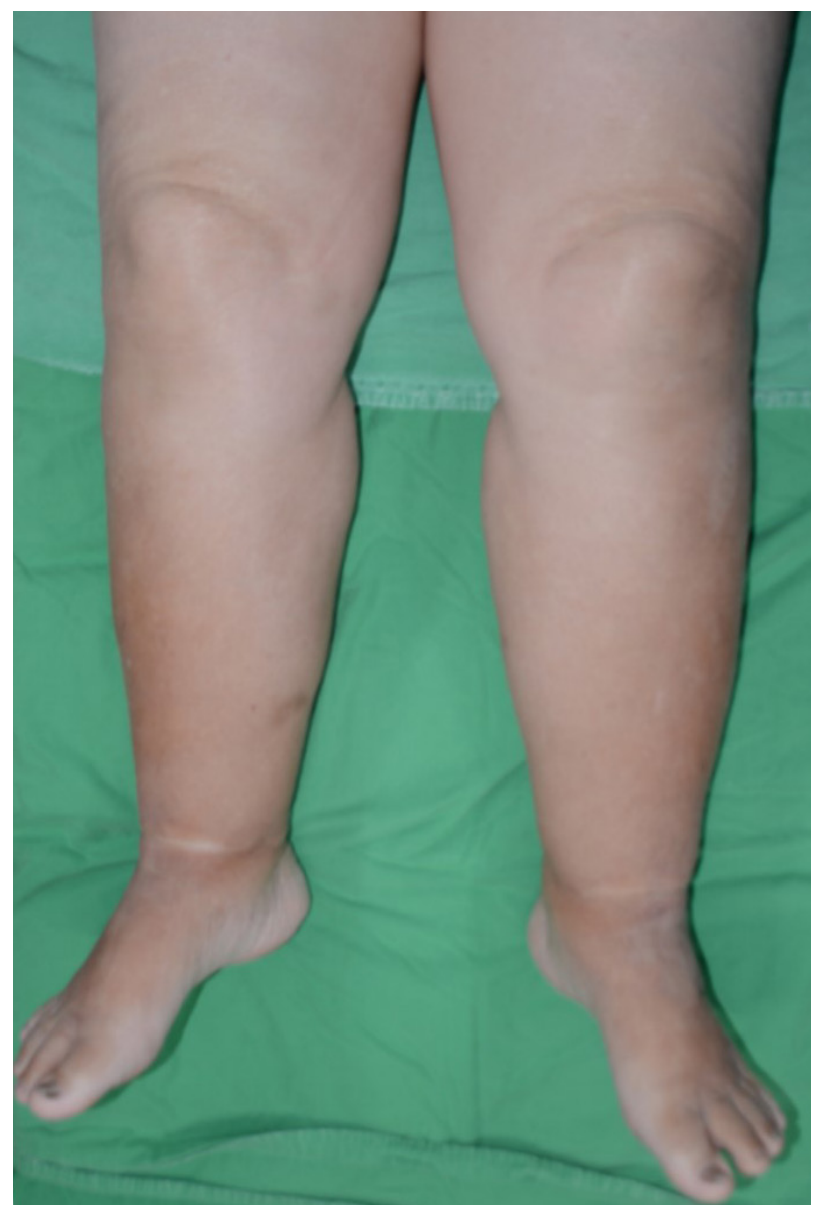

Fig. 1. Preoperative photograph.

lymphedema was confirmed on lymphoscintigraphy. Due to her significant medical history, lymph node transfer was considered but not offered, and the patient also expressed no desire to explore this possibility. We proceeded with LVA at the dorsum of the foot, distal medial leg, and mid-medial leg. Two LVAs at the foot, one LVA at the distal leg, and two LVAs at the mid-leg were performed. Lymphatic flow across the microanastomoses was demonstrated at each site. The incision sites were closed with 5/0 Vicryl and 5/0 nylon sutures over a short Penrose drain away from the anastomoses at the edge of the wound (Fig. 2). No bandage or compression garment was applied. Postoperatively, there was improved tonicity and no further episodes of cellulitis during an 18-month follow-up period (Fig. 3).

\section{DISCUSSION}

Although immediate compression garments are becoming increasingly common after LVA at many centers, we are concerned that the delicate, thin-walled vessels of microanastomoses would collapse due to mechanical compression. This would

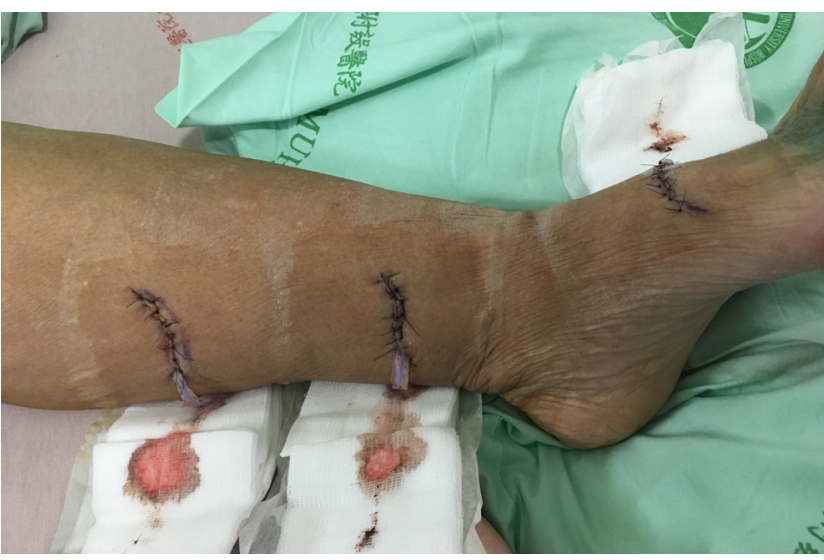

Fig. 2. Postoperative wounds. Simple interrupted sutures for wound closure over a short Penrose drain following lymphaticovenous anastomosis at multiple sites in the lower limb. The wound was covered with gauzes and Micropore tape (without a compression bandage or stocking). Skin wrinkling, indicating improved lymphedema on the morning after surgery.
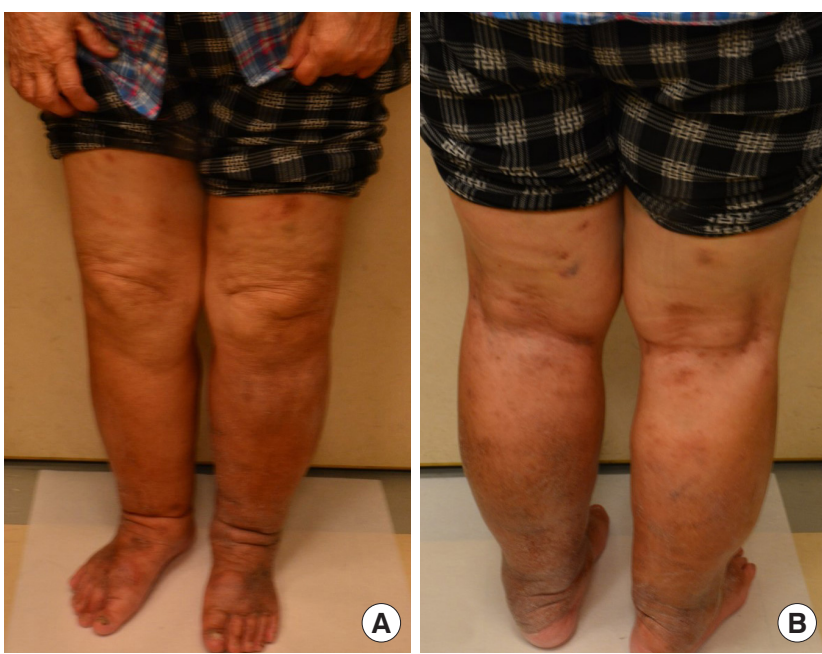

Fig. 3. Postoperative photographs. (A) Front view and (B) rear view.

stimulate coagulation within microanastomoses and cause failure of the lymphatic fluid bypass.

Previous experimental studies have shown that LVA patency decreases over time, with a study even showing no patency at 3 weeks [5]. The coagulation factors in lymph fluid have been shown to be present at levels of $50 \%$ to $60 \%$ relative to the blood plasma [6]. Furthermore, the lymph-to-plasma ratios of various coagulation factors have been reported to be between 0.05 and 0.28 [7]. Therefore, we believe that these scientific findings regarding lymph fluid justify our concerns. Since there is no published direct comparison study between immediate compression versus compression after surgery (e.g., at 1 week postoperatively), we follow a more conservative postoperative protocol. Instead of using a compression garment, we elevate the limb to 
promote lymphatic flow and to prevent stasis, thereby protecting the anastomosis site from thrombosis.

We would like to highlight first that no bandages should be placed on the wounds immediately after surgery. External compression leads to stasis of flow through the newly created LVAs, and stimulates thrombosis at the anastomotic sites in the presence of coagulation activity within the lymph fluid, as described above. We recommend wound closure with simple interrupted sutures to allow excess fluid to escape, insertion of a short Penrose drain, and gentle coverage of the incision using gauze without compression (Fig. 1). Second, ambulation should be allowed after surgery (extrinsic pump), but the operated limb should be elevated for most of the time in the initial 5 days. This will encourage lymph flow across the anastomosis without compression. We set the time of 5 days because the intima of the vessels at the anastomotic site takes 5 days to heal. Third, prophylactic antibiotics should be given because the lymphatic vessels are very fragile and are vulnerable to infection, which may again trigger thrombosis, even in cases of subclinical or mild infection.

Although a transient increase in lymphatic flow could be observed intraoperatively, it has yet to be proven that this increase in lymphatic flow continues once the incision is closed and compression is applied over the actual anastomosis and proximal to the anastomosis $[3,4]$. Another study found no difference in results whether compression was applied immediately or at 2 weeks postoperatively [8]. The practice of immediate limb compression after LVA is controversial and surgeons should exercise caution and judgment. To optimize the function of LVAs, they should be treated as ordinary microvascular anastomoses.

\section{NOTES}

\section{Conflict of interest}

No potential conflict of interest relevant to this article was reported.

\section{Ethical approval}

The study was approved by the Institutional Review Board of China Medical University Hospital (IRB No. CMUH106REC1-111[CR-1]) and performed in accordance with the principles of the Declaration of Helsinki. Written informed consent was obtained.

\section{Patient consent}

The patient provided written informed consent for the publication and the use of her images.

\section{Author contribution}

Conceptualization: JCY Chan, HC Chen. Data curation: JCY Chan, G Di Taranto, R Elia, V Amorosi, N Sitpahul, HC Chen. Formal analysis: JCY Chan, HC Chen. Methodology: JCY Chan, HC Chen. Project administration: JCY Chan, HC Chen. Visualization: JCY Chan, HC Chen. Writing - original draft: JCY Chan. Writing - review \& editing: all authors. Approval of final manuscript: all authors.

\section{ORCID}

Jeffrey C. Y. Chan https://orcid.org/0000-0001-6113-0157

Giuseppe Di Taranto https://orcid.org/0000-0002-3014-2419

Rossella Elia https://orcid.org/0000-0002-6094-5159

Vittoria Amorosi https://orcid.org/0000-0002-1190-8845

Ngamcherd Sitpahul https://orcid.org/0000-0002-4546-2255

Hung-Chi Chen https://orcid.org/0000-0002-2749-1909

\section{REFERENCES}

1. Koshima I, Nanba Y, Tsutsui T, et al. Minimal invasive lymphaticovenular anastomosis under local anesthesia for leg lymphedema: is it effective for stage III and IV? Ann Plast Surg 2004;53:261-6.

2. Winters H, Tielemans HJ, Sprangers PN, et al. Peri-operative care for patients undergoing lymphaticovenular anastomosis: a systematic review. J Plast Reconstr Aesthet Surg 2017;70:178-88.

3. Chen WF, Bowen M, Ding J. Immediate limb compression following supermicrosurgical lymphaticovenular anastomosis-is it helpful or harmful? Int Microsurg J 2018;2:1.

4. Fuse Y, Yamamoto T. Intraoperative distal compression in supermicrosurgical lymphaticovenous anastomosis for lymphedema. J Surg Oncol 2018;118:243-4.

5. Puckett CL, Jacobs GR, Hurvitz JS, et al. Evaluation of lymphovenous anastomoses in obstructive lymphedema. Plast Reconstr Surg 1980;66:116-20.

6. Fantl P, Nelson JF. Coagulation in lymph. J Physiol 1953; 122:33-7.

7. Le DT, Borgs P, Toneff TW, et al. Hemostatic factors in rabbit limb lymph: relationship to mechanisms regulating extravascular coagulation. Am J Physiol 1998;274:H769-76.

8. Yang JC. Reply to "Intraoperative distal compression in supermicrosurgical lymphaticovenous anastomosis for lymphedema”. J Surg Oncol 2018;118:245. 